\title{
Prophylaxis of Early Ventricular Fibrillation by Inhibition of Acylcarnitine Accumulation
}

\author{
Peter B. Corr, Michael H. Creer, Kathryn A. Yamada, Jeffrey E. Saffitz, and Burton E. Sobel \\ Cardiovascular Division, Department of Medicine and Department of Pharmacology, \\ Washington University School of Medicine, St. Louis, Missouri 63110
}

\begin{abstract}
Hypoxia in isolated myocytes results in accumulation of longchain acylcarnitines (LCA) in sarcolemma. Inhibition of carnitine acyltransferase I (CAT-I) with sodium 2-[5-(4-chlorophenyl)-pentyl]-oxirane-2-carboxylate (POCA) prevents both the accumulation of LCA in the sarcolemma and the initial electrophysiologic derangements associated with hypoxia. Another amphiphilic metabolite, lysophosphatidylcholine (LPC), accumulates in the ischemic heart in vivo, in part because of inhibition of its catabolism by accumulating LCA. It induces electrophysiologic alterations in vitro analogous to early changes induced by ischemia in vivo. The present study was performed to determine whether POCA could prevent accumulation of both LCA and LPC induced by ischemia in vivo and if so, whether attenuation of early arrhythmogenesis would result. LAD coronary artery occlusions were induced for $5 \mathrm{~min}$ in chloralose-anesthetized cats. Coronary occlusion in untreated control animals elicited prompt, threefold increases of LCA (73 \pm 8 to $286 \pm 60 \mathrm{pmol} / \mathrm{mg}$ protein) and twofold increase of LPC (3.3 \pm 0.4 to $7.5 \pm 0.9 \mathrm{nmol} / \mathrm{mg}$ protein) selectively in the ischemic zone, associated with ventricular tachycardia (VT) or ventricular fibrillation (VF) occurring within the 5-min interval before acquisition of myocardial samples in $64 \%$ of the animals. POCA prevented the increase of both LCA and LPC. It also prevented the early occurrence of VT or VF (within 5 min of occlusion) in all animals studied. The antiarrhythmic effect of POCA was not attributable to favorable hemodynamic changes or to changes in myocardial perfusion measured with radiolabeled microspheres. Thus, inhibition of CAT-I effectively reduced the incidence of lethal arrhythmias induced early after the onset of ischemia. Accordingly, pharmacologic inhibition of this enzyme provides a promising approach for prophylaxis of sudden cardiac death, that typically occurs very soon after the onset of acute ischemia, in man.
\end{abstract}

\section{Introduction}

Amphipathic metabolites, including lysophosphoglycerides and long-chain acylcarnitines have been shown to accumulate in the ischemic heart. They exhibit several properties that may contribute to arrhythmogenesis induced early after the onset of

Address reprint requests to Dr. Corr, Cardiovascular Division, Washington University School of Medicine, 660 South Euclid Avenue, Box 8086, St. Louis, MO 63110.

Received for publication 22 July 1988 and in revised form 15 September 1988.

J. Clin. Invest.

(c) The American Society for Clinical Investigation, Inc.

$0021-9738 / 89 / 03 / 0927 / 10 \quad \$ 2.00$

Volume 83, March 1989, 927-936 ischemia judging from results in vitro (1-6). This study was designed to determine whether their accumulation could be inhibited and if so whether the inhibition resulted in protection against early malignant ventricular arrhythmias induced by ischemia in vivo.

Long-chain acylcarnitines, one class of amphipathic metabolites, activate calcium channels in cardiac $(7,8)$ and smooth muscle myocytes $(8,9)$. Accordingly, they may potentiate the increase in cytosolic calcium associated with arrhythmogenesis in ischemic myocardium (10). Increases of longchain acylcarnitines in sarcolemma are induced by hypoxia judging from EM autoradiographic measurements of cells prelabeled with $\left[{ }^{3} \mathrm{H}\right]$ carnitine (11). Inhibition of carnitine acyltransferase I precludes the accumulation of long-chain acylcarnitines and attenuates the electrophysiologic derangements induced by hypoxia in myocytes in vitro (11).

Accumulation of long-chain acylcarnitines in sarcolemma is paralleled by an increase in myocytic surface $\alpha_{1}$-adrenergic receptors (12) and associated receptor-mediated generation of inositol trisphosphate $\left(\mathrm{IP}_{3}\right)^{1}$ (13). Alpha ${ }_{1}$-adrenergic blockade is antiarrhythmic in ischemic and reperfused myocardium (14). Thus, inhibition of carnitine acyltransferase I may be antiarrhythmic not only by precluding direct, deleterious electrophysiologic effects otherwise induced by long-chain acylcarnitines but also indirectly by attenuating $\alpha_{1}$-adrenergic receptor-mediated influences on arrhythmogenesis in the ischemic heart.

Antiarrhythmic effects of inhibition of carnitine acyltransferase I may be manifestations of a third process as well. Lysophosphatidylcholine (LPC), an amphiphile structurally similar to long-chain acylcarnitine, accumulates in ischemic myocardium $(1,4,15,16)$. Incorporation of LPC into the sarcolemma of normoxic myocytes (1-2 mol \%), documented by EM autoradiography, is associated with reversible electrophysiologic derangements $(17,18)$ analogous to those seen in the ischemic heart in vivo. Because the activity of catabolic enzymes responsible for the degradation of LPC exceeds the activity of synthetic enzymes under physiologic conditions, accumulation of LPC in the ischemic heart appears to reflect inhibition of catabolism (1). At concentrations that prevail in ischemic myocardium, long-chain acylcarnitines competitively inhibit two key catabolic enzymes, lysophospholipase-transacylase and lysophospholipase $(19,20)$. Accordingly, inhibition of the accumulation of long-chain acylcarnitines by inhibition of

1. Abbreviations used in this paper: FAME, fatty acid methyl ester; FID, flame ionization detector; $\mathrm{IP}_{3}$, inositol trisphosphate; LAD, left anterior descending; LPC, lysophosphatidylcholine; LV, left ventricle; PCA, perchloric acid; POCA, sodium 2-[5-(4-chlorophenyl)-pentyl]oxirane-2-carboxylate; TPR, total peripheral resistance; VF, ventricular fibrillation; VT, ventricular tachycardia. 
carnitine acyltransferase I may attenuate accumulation of LPC in ischemic myocardium thereby reducing arrhythmogenicity.

This study was performed to determine whether inhibition of carnitine acyltransferase I reduces the accumulation of long-chain acylcarnitines and LPC otherwise occurring with ischemia in vivo and whether inhibition prevents early ventricular arrhythmias including fibrillation otherwise resulting immediately after the ischemic insult.

\section{Methods}

Animal preparations. Adult cats were anesthetized with ketamine $\mathrm{HCl}$ $(12.5 \mathrm{mg} / \mathrm{kg})$ and $\alpha$-chloralose $(75 \mathrm{mg} / \mathrm{kg})$, intubated, and ventilated with a Harvard respirator (Harvard Apparatus Co., Natick, MA). Muscular relaxation was maintained with intravenous injections of decamethonium bromide $(0.25 \mathrm{mg} / \mathrm{kg})$. Catheters were inserted into the femoral artery and vein. A recorder (model 260; Gould-Brush, Inc., Oxnard, CA) was used to monitor a lead II surface electrocardiogram and systemic arterial pressure. In several animals, left ventricular pressure was recorded simultaneously via a catheter inserted into the left ventricle through the apex. Mean and pulsatile aortic flow were measured with the use of a calibrated flow probe $(5 \mathrm{~mm})$ and flowmeter with electronic zero (Narco Bio-Systems, Inc., Houston, TX). Total peripheral resistance (TPR) was expressed in PR units calculated as follows: TPR (PR units) $=$ (mean arterial pressure $(\mathrm{mmHg}) \times 60$ $\mathrm{s} / \mathrm{min}) \div$ cardiac output $(\mathrm{ml} / \mathrm{min})$. Body temperature was maintained at $37^{\circ} \mathrm{C}$ via a thermostatic esophageal probe controlling an infrared lamp. A left thoracotomy was performed by excision of ribs two through four, a pericardial cradle was constructed, and the left anterior descending (LAD) coronary artery was isolated at its bifurcation from the main left trunk immediately proximal to all branch points. Care was taken to avoid the pericoronary nerve coursing parallel to the artery. A 3-0 cotton suture was placed under the vessel, and polyethylene tubing was threaded around the suture. Coronary occlusion was instituted by advancing the polyethylene tubing to the artery and clamping it in place. In selected experiments, sodium 2-[5-(4-chlorophenyl)-pentyl]-oxirane-2-carboxylate (POCA), $7.3 \mathrm{mg} / \mathrm{kg}$ i.v. was administered $10 \mathrm{~min}$ before the onset of ischemia, a time interval required for uptake and inhibition of carnitine acyltransferase $I(11,12)$. In those experiments in which regional blood flow and hemodynamic evaluations were performed, reperfusion was accomplished by releasing the clamp and retracting the polyethylene tubing. Samples of tissue from normal and ischemic zones of cat hearts in vivo were obtained by high-speed, suction drill biopsy and were fast frozen in liquid $\mathrm{N}_{2}$. Ischemic zones were readily identified by epicardial cyanosis in the regions supplied by the occluded coronary artery and sampled after the onset of proximal LAD coronary occlusion as previously described (4). Transmural biopsies were obtained either prior to ischemia in control, nonischemic animals or $5 \mathrm{~min}$ after ischemia in animals who did not develop ventricular fibrillation. In animals which developed ventricular fibrillation, the biopsies were obtained immediately. Due to the transmural nature of the biopsies, the experiments were terminated immediately thereafter. The frozen tissue was pulverized in a stainless steel mortar and pestle cooled in liquid $\mathrm{N}_{2}$ and stored at $-70^{\circ} \mathrm{C}$. Biochemical analyses were performed within two weeks.

Regional myocardial blood flow. In selected animals, regional myocardial blood flow was determined as follows. A femoral arterial catheter, other than the one used for pressure monitoring, was advanced to the descending aorta for withdrawal of reference blood samples. A left atrial catheter held in place with a purse string suture was used for administration of microspheres. Before and after each experiment, an infusion pump (model 351; Sage Instruments, Cambridge, MA) was calibrated with respect to flow rates. Carbonized microspheres $(9 \mu \mathrm{m})\left(3 \mathrm{M}\right.$ Company, St. Paul, MN) labeled with ${ }^{141} \mathrm{Ce},{ }^{51} \mathrm{Cr}$, ${ }^{85} \mathrm{Sr}$, or ${ }^{46} \mathrm{Sc}$ in saline were sonicated for at least $10 \mathrm{~min}$ before injection (random order) followed by mechanical disaggregation within a pair of syringes and stopcocks. Microspheres were injected before LAD coro- nary occlusion, 3-4 min after coronary occlusion, 15-17 min after reperfusion, and again 3-4 min after a second coronary occlusion. POCA $(7.3 \mathrm{mg} / \mathrm{kg}$ i.v.) was administered $10 \mathrm{~min}$ after the onset of reperfusion. Approximately $4.2 \times 10^{5}$ to $2.1 \times 10^{6}$ microspheres were administered by bolus injection into the left atrium followed by a saline flush in each case. Arterial blood was withdrawn at a rate of 3 $\mathrm{ml} / \mathrm{min}$ before, during, and $45 \mathrm{~s}$ after injection of the spheres. Injections did not alter blood pressure or heart rate discernibly.

Blood samples were vortexed and divided into four equal aliquots. After excision of the heart, the entire left ventricle was dissected clean, opened along the posterior septum, and divided into 22 endocardial and epicardial tissue samples and two papillary muscles for assay of radioactivity in an universal gamma counter (LKB Wallac 1282 Compugamma; LKB Instruments, Inc., Gaithersburg, MD). Samples within the perfusion territory of the LAD coronary artery comprising $\sim 40 \%$ of the left ventricular mass were classified as ischemic; the remaining samples were classified as nonischemic. Regional blood flow was calculated according to Heymann et al. (21) and expressed as $\mathrm{ml}-\mathrm{min}^{-1}-\mathrm{g}^{-1}$.

Chemical reagents. [acetyl- $1-{ }^{14} \mathrm{C}$ ]Acetyl-coenzyme A was purchased from New England Nuclear (Boston, MA) and 1-heptadecanoyl-2-lysophosphatidylcholine (17:0 LPC) from Sigma Chemical Co. (St. Louis, MO). The 17:0 LPC was purified by HPLC and the purified product derivatized to form fatty acid methyl esters (FAME), which were subsequently analyzed by capillary GC as described below. The purified product contained > 99\% 17:0 LPC with no other LPC molecular species detectable. The concentration of 17:0 LPC in the final purified product was determined by assay of lipid phosphorus (22) after preliminary ashing in $1.2 \mathrm{M} \mathrm{Mg}\left(\mathrm{NO}_{3}\right)_{2}$ in ethanol (23). POCA was a gift from Dr. H. P. O. Wolf, ByK Gulden, Konstanz, FRG. Crystalline POCA was stored in a dark brown vial at $-10^{\circ} \mathrm{C}$. The quantity of drug required for each specific animal $(7.3 \mathrm{mg} / \mathrm{kg})$ was dissolved in $2.0 \mathrm{ml}$ of distilled water and used within $2 \mathrm{~h}$ of preparation. Organic solvents were of HPLC grade. All other chemical reagents were of the highest analytical grade available.

Quantification of $L P C$. For quantification of the content of LPC in myocardial tissue, $50-100 \mathrm{mg}$ of frozen myocardium was transferred to a Duall glass homogenizer tube, and the tissue was homogenized in $3 \mathrm{ml}$ of methanol/distilled water $(1: 1, \mathrm{vol} / \mathrm{vol})$. For every $1 \mathrm{mg}$ of frozen myocardium in the homogenate, $0.5 \mathrm{nmol}$ of 17:0 LPC was added as an internal standard. The sample phospholipids were extracted twice with chloroform $\left(\mathrm{CHCl}_{3}\right)$ and methanol at a temperature of $0-4^{\circ} \mathrm{C}(24)$. The chloroform layers from each extraction step were combined and evaporated to dryness under a stream of dry $\mathrm{N}_{2}$ gas. The

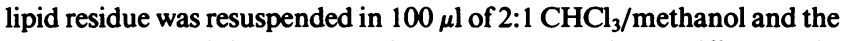
sample phospholipids separated into classes according to differences in polar headgroup composition by HPLC on a silica-based cation-exchange column ( $10 \mu \mathrm{m}$, Partisil-SCX; Whatman, Inc., Clifton, NJ) by isocratic elution using a ternary solvent system composed of acetonitrile/methanol/water (400:100:28) as previously described (25). Examples of results of the separative procedure are shown in Fig. 1.

With the separation method used, the acidic phospholipid cardiolipin (diphosphatidylglycerol) elutes as a broad peak and accounts for $10-20 \%$ of the total lipid phosphorus present in the LPC fraction. To remove cardiolipin, the LPC fraction was evaporated to dryness under a stream of dry $\mathrm{N}_{2}$ gas, resuspended in $500 \mu \mathrm{l}$ of $1: 1 \mathrm{CHCl}_{3} /$ methanol, and applied to a solid-phase extraction tube containing a silica-based anion-exchange packing (Supelclean LC-SAX; Supelco, Inc., Bellefonte, PA) that had been washed previously with $5 \mathrm{ml}$ of $1: 1 \mathrm{CHCl}_{3} /$ methanol. After the extraction tube had been washed with an addi-

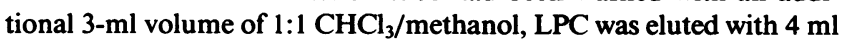
of methanol. After collection, the methanol eluate was evaporated to dryness under a stream of dry $\mathrm{N}_{2}$ gas and the LPC hydrolyzed for 90 min in $1 \mathrm{~N} \mathrm{HCl}$ in methanol (Supelco) at $90^{\circ} \mathrm{C}$ to form fatty acid methyl esters.

FAME products were recovered by pentane extraction and separated by gas chromatography (GC) on a $60 \mathrm{~m} \times 0.25 \mathrm{~mm}$ fused-silica capillary column coated with SP 2330 (Supelco) at $170^{\circ} \mathrm{C}$ (isothermal) 

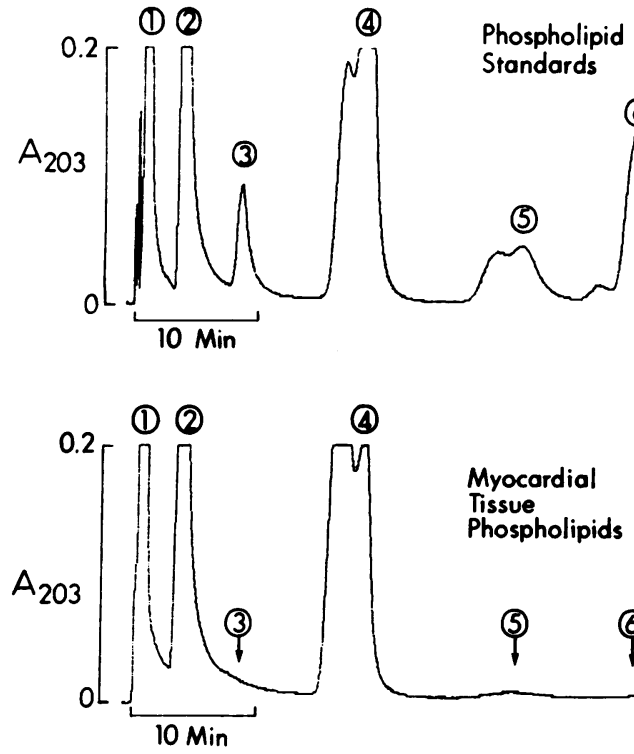

(4)

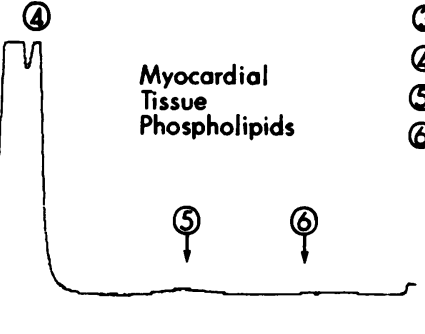

Figure 1. HPLC tracing of the separation of phospholipid standards (top) and myocardial tissue phospholipids recovered by chloroform/ methanol extraction (bottom). Approximately $0.5-1.0 \mu \mathrm{mol}$ of total phospholipid dissolved in $50 \mu \mathrm{l}$ of chloroform/methanol (2:1, vol/ vol) were injected onto a 10- $\mu \mathrm{m}$ Partisil SCX column. Individual phospholipid classes were separated using isocratic elution with a ternary mobile phase comprised of acetonitrile/methanol/water $(400: 100: 28, \mathrm{vol} / \mathrm{vol})$ at a flow rate of $2.5 \mathrm{ml} / \mathrm{min}$ and operating pressure of 1,000 psi. Phospholipids eluting from the column that contained unsaturated esterified fatty acids were detected by measurement of ultraviolet absorption at $203 \mathrm{~nm}\left(\mathrm{~A}_{203}\right)$. SF, solvent front; PE, phosphatidylethanolamine; LPE, lysophosphatidylethanolamine; PC, phosphatidylcholine; SPH, sphingomyelin; and LPC, lysophosphatidylcholine.

as previously described (26). Individual FAME products were quantified with a flame ionization detector. A representative example of a GC separation of the FAME products derived from LPC is shown in Fig. 2. The quantity of individual FAME species was calculated using the following formula: Amt $(x)=[$ Area (x)/Area (IS) $] \times[M W(I S) / M W$ $(x)] \times$ Amt (IS) where Amt $(x)=$ amount of FAME, Area $(x)=$ integrated area for flame-ionization detector (FID) response to FAME, Area (IS) $=$ integrated area for FID response to 17:0 FAME internal standard (IS), MW (IS) = molecular weight of 17:0 FAME internal standard, MW (x) = molecular weight of FAME, and Amt (IS) $=$ amount of 17:0 LPC internal standard added to the tissue sample before homogenization ( $\mathrm{nmol} / \mathrm{mg}$ frozen tissue). The detector response factor for different FAME species of selected chain lengths $\left(\mathrm{C}_{16}-\mathrm{C}_{20}\right)$ is proportional to their molecular weights (26). Because $1 \mathrm{~mol}$ of FAME is produced for each mole of LPC, the total LPC content in the tissue is determined from the sum (in moles) of the amounts of individual FAME species.

Quantification of carnitine. Total carnitine, long-chain acylcarnitine, total acid soluble carnitine and free carnitine fractions in tissue samples were separated based on the insolubility of long-chain acylcarnitine esters in 7\% perchloric acid (PCA) and the use of base-catalyzed hydrolysis of esterified carnitine as described previously (27). Frozen myocardium (100-150 mg) was homogenized in $3 \mathrm{ml}$ of $7 \%$ PCA, and a 300- $\mu$ l aliquot of the homogenate was removed for assay of the total carnitine fraction. The remaining homogenate was centrifuged at $10,000 \mathrm{~g}$ for $30 \mathrm{~min}$, the supernate removed, the pellet washed twice with $3 \mathrm{ml}$ of $7 \% \mathrm{PCA}$, and resuspended in $500 \mu \mathrm{l}$ of distilled water for assay of the long-chain acylcarnitine fraction. The supernate and 7\% PCA washes were combined and one-half of the total volume was used for assay of total acid soluble carnitine. The remainder was used

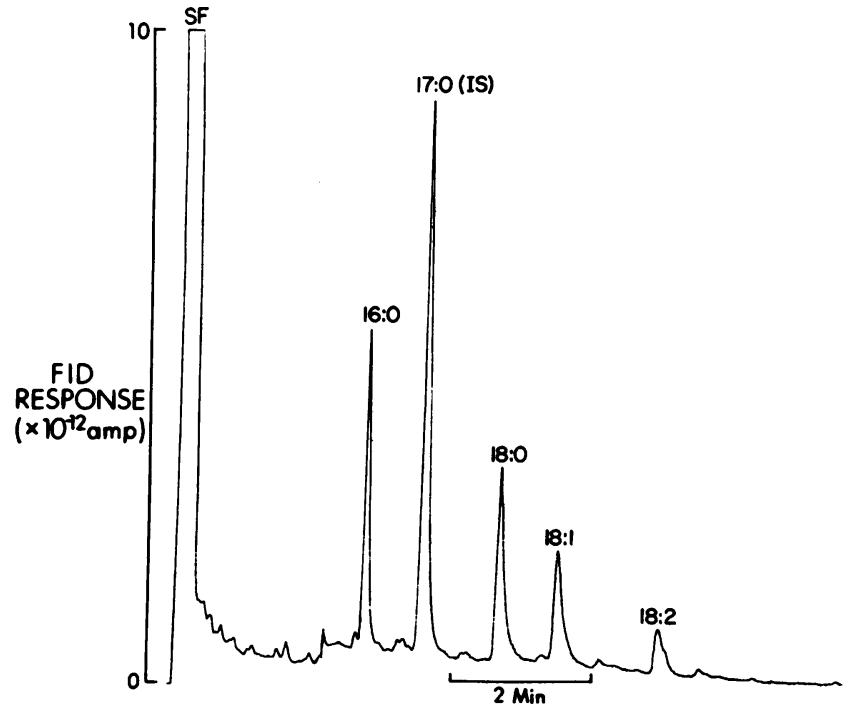

Figure 2. Gas chromatographic tracing of the separation of FAME products derived from the LPC fraction after HPLC separation and removal of cardiolipin using a silica based, strong anion exchange (SAX) column. The FAME products were dissolved in $0.5 \mu \mathrm{l}$ of pentane, injected onto a 60 meter fused silica capillary column coated with SP-2330, and eluted at $170^{\circ} \mathrm{C}$ (isothermal) with helium as a carrier gas at a flow rate of $0.3 \mathrm{ml} / \mathrm{min}$. Individual FAME species were detected with a FID. Peaks corresponding to the methyl esters of palmitic (16:0), heptadecanoic (internal standard, IS, 17:0), stearic (18:0), oleic (18:1), and linoleic (18:2) fatty acids were identified based on comparison of their relative retention times with those of a mixture of standards containing the methyl esters of C16-C20 fatty acids.

for assay of free carnitine. A $200-\mu \mathrm{l}$ volume of $5 \mathrm{M} \mathrm{KOH}$ was added to the total carnitine, long-chain acylcarnitine, and total acid soluble carnitine fractions, which were then hydrolyzed for $90 \mathrm{~min}$ at $70^{\circ} \mathrm{C}$ in a water bath. Fractions were neutralized by the addition of 0.5 to 1.0 $\mathrm{ml}$ of $1 \mathrm{M}$ Hepes buffer $(\mathrm{pH}=8)$ and additional 7\% PCA or $5 \mathrm{M} \mathrm{KOH}$ as needed to adjust the $\mathrm{pH}$ to $7-8$. The $\mathrm{KClO}_{4}$ precipitate remaining after neutralization was removed by centrifugation of the samples at $10,000 \mathrm{~g}$ for $30 \mathrm{~min}$ after which the precipitates were washed with an additional $1-\mathrm{ml}$ volume of distilled water.

Free (i.e., nonesterified) carnitine was measured in each fraction using a modification of the radioenzymatic method of McGarry and Foster (28). Assays were performed in disposable glass culture tubes $(13 \times 100 \mathrm{~mm})$. The reaction mixture contained, in a volume of 1.0 $\mathrm{ml}, 120 \mu \mathrm{mol}$ of Hepes buffer ( $\mathrm{pH}=7.4$ ); $1.25 \mu \mathrm{mol}$ EDTA; $2.0 \mu \mathrm{mol}$ of $N$-ethylmaleimide; $5.0 \mathrm{nmol}(0.04 \mu \mathrm{Ci})$ of $\left[{ }^{14} \mathrm{C}\right]$ acetyl-CoA; and standards or samples to be analyzed containing $50-2,500 \mathrm{pmol}$ of free carnitine. The reaction was initiated by the addition of $50 \mu \mathrm{l}$ of a solution prepared by diluting $200 \mu \mathrm{l}$ of carnitine acetyltransferase (10 $\mathrm{mg}$ protein [ 800 units]) in $2 \mathrm{ml}$ of $2.9 \mathrm{M}$ ammonium sulfate (Sigma) and $150 \mu \mathrm{l}$ of $0.83 \mathrm{M}$ Hepes buffer $(\mathrm{pH}=7.4)$ to a total volume of 2.0 $\mathrm{ml}$ with distilled water. Samples were incubated at room temperature for $60 \mathrm{~min}$. After incubation, $750 \mu \mathrm{l}$ of the reaction mixture were transferred to a $13 \times 100 \mathrm{~mm}$ serum separator tube with a 5- $\mu \mathrm{m}$ filter (Fisher Scientific, Pittsburgh, PA) that contained 700-800 mg of acidwashed Dowex AG1-X8 anion exchange resin (Bio-Rad Laboratories, Richmond, CA) for removal of unreacted $\left[{ }^{14} \mathrm{C}\right]$ acetyl-CoA from the reaction mixture. The serum separator tubes were placed inside a 13 $\times 100 \mathrm{~mm}$ glass culture tube to form an airtight seal thereby allowing the reaction mixtures to filter through the anion exchange resin when the serum separator tube was withdrawn partially. The resin bed was 
washed with an additional $300-\mu$ l volume of distilled water and the serum separator tube was withdrawn completely and discarded. $750 \mu \mathrm{l}$ of the filtrate containing the $\left[{ }^{14} \mathrm{C}\right]$ acetylcarnitine reaction product were added to $10 \mathrm{ml}$ of Aquasol (New England Nuclear) and radioactivity in the samples counted with a liquid scintillation counter (Beckman LS 3801; Beckman Instruments, Inc., Fullerton, CA). Sample carnitine concentration was determined from the least-squares linear regression curve from samples of carnitine standards containing 50-2500 pmol of carnitine.

Recovery of free carnitine added to the tissue before homogenization was $97 \pm 6 \%(n=6)$. Recovery of long-chain acylcarnitines (assessed with palmitoyl-L-carnitine added to the tissue before homogenization) was $92 \pm 12 \%(n=8)$.

Results were normalized according to the protein content of the tissue samples measured as described by Markwell (29) with the use of lyophilized bovine serum albumin (Bio-Rad Laboratories) as the protein standard.

\section{Results}

Arrhythmias. Data from animals subjected to coronary artery occlusion for $5 \mathrm{~min}$ are summarized in Fig. 3. In animals not given POCA before the 5-min interval of ischemia, the incidence of immediate ventricular tachycardia (VT) and/or ventricular fibrillation (VF), i.e., within $5 \mathrm{~min}$, was $64 \%(9 / 14$ animals). VT was defined as greater than five repetitive premature ventricular complexes (PVCs) with a QRS morphology different from sinus beats and a width greater than sinus beats associated with a decrease in systemic arterial pressure. VF was defined as chaotic ventricular electrical activity not associated with the development of any systemic arterial pressure pulse. In untreated control ischemic animals that did not develop VF (8/14 animals), the frequency of PVCs during the 5-min interval of ischemia was $191 \pm 83$ (mean \pm SEM). In contrast, in animals treated with POCA (7.3 mg/kg body wt i.v.) $10 \mathrm{~min}$ before the onset of ischemia, the incidence of immediate VT or VF (within $5 \mathrm{~min}$ ) was $0 \%$ (0/8 animals) and the frequency of PVCs during the 5-min interval of ischemia before sampling myocardium was $20 \pm 11$ (mean \pm SEM, $P=0.06$ ). Thus, POCA prevented early VT and VF and reduced the frequency of PVCs during a 5-min interval of ischemia. POCA did not exert significant inotropic or chronotropic effects. There were no significant differences in mean arterial pressure (134 \pm 15 vs. $146 \pm 4 \mathrm{mmHg}$ ) or heart rate $(198 \pm 9 \mathrm{vs} .203 \pm 9$ beats $/ \mathrm{min})$ with sinus rhythm whether or not treatment with POCA had been implemented. Changes in the response to ischemia of systemic arterial pressure and heart rate associated with sinus rhythm did not differ in animals with compared with those without treatment with POCA (see below). As demonstrated pre-
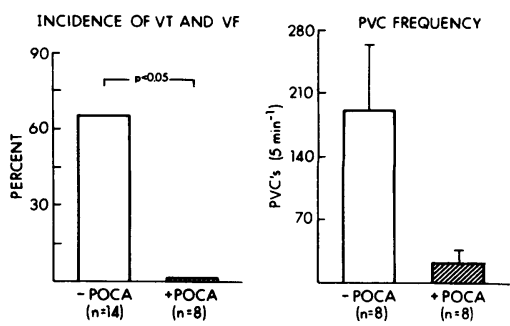

\begin{abstract}
Figure 3. Incidence of ventricular tachycardia (VT) and ventricular fibrillation (VF) (left panel) and the frequency of premature ventricular complexes (PVC frequency) (right panel) during the 5-min interval of ischemia in
\end{abstract} untreated animals (-POCA group) and in animals given POCA (7.3 $\mathrm{mg} / \mathrm{kg}$, IV) $10 \mathrm{~min}$ before coronary occlusion (+POCA group). VF was seen in six untreated animals and VT was seen in seven untreated animals during the first $5 \mathrm{~min}$ of ischemia. In four untreated animals, both VT and VF occurred.

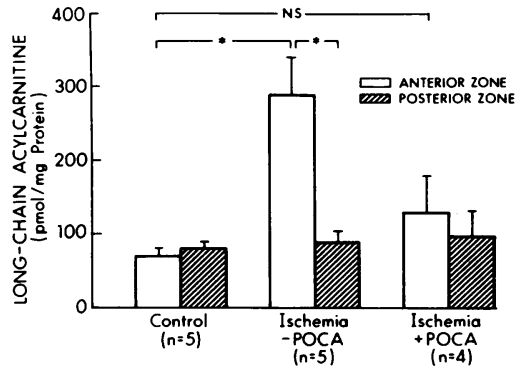

Figure 4. Values for long-chain acylcarnitines in the anterior (ischemic) zone and posterior (normal) zone in sham operated control animals without ischemia (control), at the end of the 5-min interval of ischemia induced by occlusion of

the LAD coronary artery in untreated animals (Ischemia - POCA), and in animals given POCA 10 min before coronary occlusion (Ischemia + POCA). ${ }^{*} P<0.05$ for comparison of group means by ANOVA.

viously with intracellular transmembrane recordings from isolated myocytes, POCA has no direct electrophysiologic effects (11).

Acylcarnitine content. In five control animals without ischemia, the coronary artery was isolated and biopsies were obtained after sham operation. Long-chain acylcarnitine values in these sham operated control animals were virtually the same in anterior and posterior regions of the left ventricular wall (Fig. 4). In animals subjected to ischemia for $5 \min (n=5)$, long-chain acylcarnitines increased 3.5-fold in the anterior, ischemic zone compared with values in sham operated controls, but values in the posterior (normal) zone did not differ significantly from those in corresponding zones in sham-operated controls. In contrast, pretreatment of animals with POCA significantly attenuated the increase of long-chain acylcarnitines in anterior, ischemic zones compared with values in untreated animals (Fig. 4). Concentrations of long-chain acylcarnitines in the anterior, ischemic zones of animals treated with POCA were not significantly greater than those in anterior zones of nonischemic control animals (Fig. 4). Values in nonischemic posterior zones of animals treated with POCA did not differ significantly from those in corresponding zones of controls or in posterior zones of animals that were not given POCA prior to induction of anterior wall ischemia. Thus, treatment with POCA prevented the accumulation of longchain acylcarnitines in ischemic myocardium without affecting long-chain acylcarnitine content in nonischemic tissue.

Concentrations of total carnitine, short-chain acylcarnitine, primarily acetylcarnitine, and free carnitine in anterior (ischemic) zones and posterior (normal) zones of controls and animals subjected to ischemia with or without prior treatment with POCA are shown in Table I. No significant differences were noted. The variability of concentration of total tissue carnitine in all three groups is likely to be attributable to differences in rates of endogenous synthesis and dietary intake of carnitine. Total carnitine in anterior and posterior zones of all three groups averaged $10.7 \mathrm{nmol} / \mathrm{mg}$ protein or $1.6 \mu \mathrm{mol} / \mathrm{g}$ wet wt of tissue, a value comparable to that in hearts from other species (30).

The content of free carnitine did not differ significantly in anterior and posterior zones in any of the three groups (Table I). Ischemia resulted in a modest increase of short-chain acylcarnitines in anterior, ischemic zones in animals that had not been treated with POCA compared with values in corresponding zones of sham-operated controls, but the increase was not statistically significant. Similar results were observed in animals treated with POCA in which ischemia elicited a nonsig- 
Table I. Total Carnitine, Free Carnitine and Short-chain Acylcarnitine in Control and Ischemic Hearts

\begin{tabular}{|c|c|c|c|c|c|c|}
\hline & \multicolumn{3}{|c|}{ Anterior zone } & \multicolumn{3}{|c|}{ Posterior zone } \\
\hline & TC & SCA & FC & TC & SCA & FC \\
\hline & \multicolumn{3}{|c|}{ nmol/mg protein } & \multicolumn{3}{|c|}{ nmol/mg protein } \\
\hline Control $(n=5)$ & $8.23 \pm 1.25$ & $1.36 \pm 0.39$ & $6.49 \pm 1.30$ & $9.38 \pm 1.74$ & $1.59 \pm 0.27$ & $7.79 \pm 1.54$ \\
\hline Ischemia $(n=5)$ & $9.05 \pm 0.97$ & $2.20 \pm 0.21$ & $7.09 \pm 1.03$ & $8.26 \pm 0.88$ & $1.40 \pm 0.20$ & $7.06 \pm 0.69$ \\
\hline Ischemia with POCA $(n=4)$ & $11.80 \pm 2.83$ & $2.39 \pm 0.50$ & $9.42 \pm 2.31$ & $12.10 \pm 2.50$ & $1.34 \pm 0.36$ & $10.90 \pm 2.21$ \\
\hline
\end{tabular}

Values are means \pm SEM. No significant differences were found in any of the intergroup comparisons by ANOVA or by comparison of values in anterior compared with posterior regions in the same animals by paired Student's $t$ test. TC, total carnitine; SCA, short-chain acylcarnitine; FC, free carnitine.

nificant increase in short-chain acylcarnitine content in anterior zones compared with corresponding values in sham-operated controls. Effects of ischemia on long-chain acylcarnitines were therefore selective compared with effects on total, free, or short-chain acylcarnitines. Because long-chain acylcarnitines constitute only $1-2 \%$ of the total carnitine pool in myocardial tissue, even a two- to threefold increase would not necessarily be associated with a significant decline of free carnitine especially in view of the relatively wide range of free and total carnitine values in myocardium.

LPC content. LPC content in anterior zones of hearts of sham operated controls averaged $3.31 \mathrm{nmol} / \mathrm{mg}$ protein, i.e., $\sim 2.2 \%$ of total tissue phospholipid (Fig. 5). This value is comparable to values reported previously for cat myocardium and hearts of other mammalian species $(4,23,31,32)$. After 5 min of ischemia, LPC in anterior (ischemic) zones increased over twofold to $7.46 \mathrm{nmol} / \mathrm{mg}$ protein, a $125 \%$ (2.25-fold) increase compared with values in the anterior zones of controls and a $79 \%$ (1.8-fold) increase compared with values in nonischemic, posterior zones in the same hearts. LPC concentration in posterior zones of hearts of animals subjected to ischemia was comparable to that in corresponding regions of hearts from control, nonischemic hearts.

Treatment of animals with POCA 10 min before the onset of ischemia prevented the increase of LPC in anterior zones

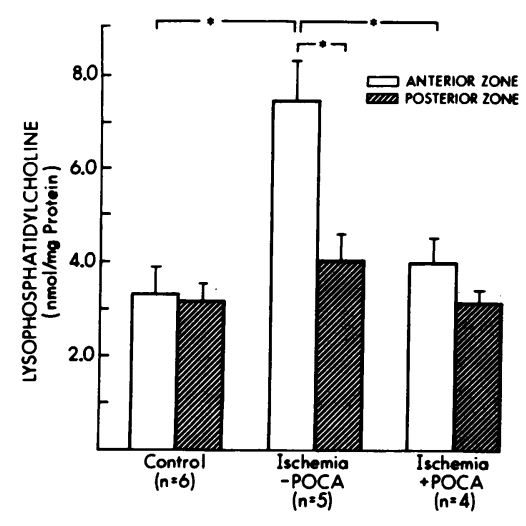

Figure 5. Values for lysophosphatidylcholine in the anterior (ischemic) zone and posterior (normal) zone in sham operated control animals without ischemia (control), at the end of the 5-min interval of ischemia induced by occlusion of the LAD coronary artery in untreated animals (Ischemia - POCA), and in animals given POCA 10 min before coronary occlusion (Ischemia + POCA). ${ }^{*} P<0.05$ for comparison of group means by ANOVA. without affecting LPC content in posterior (normal) zones (Fig. 5). LPC content in anterior and posterior zones of animals treated with POCA was not significantly different from that in corresponding regions of controls.

Because substantial remodeling of the fatty acid composition of phospholipids occurs with prolonged intervals of ischemia (33), we characterized the fatty acid composition of LPC in ischemic myocardium. The distribution of LPC molecular species in each of the three groups of animals are shown in Table II. Most of the esterified fatty acids in LPC in tissue from controls were comprised of palmitate (16:0) and stearate (18:0). Although ischemia resulted in a net increase of LPC, the distribution of esterified fatty acids in LPC was not altered. Accordingly, there appeared to be no species selectivity for the increased production of LPC in ischemic hearts. Although treatment with POCA prevented the increase of LPC induced by ischemia, the distribution of LPC molecular species did not change. The posterior zones did not exhibit significant differences in fatty acid profiles of LPC in any of the three groups evaluated (Table II). There were no significant differences in the percentages of nonidentified peaks (7-12\%) in anterior or posterior zones in tissue from the three groups.

Cardiolipin contamination of the LPC fraction obtained after HPLC separation of the major phospholipid classes could falsely elevate the levels of LPC. However, with the SAX column, it was possible to effectively remove cardiolipin from the LPC fraction after HPLC separation. LPC accounts for $\sim 97$ mol\% of the total phospholipid in the eluate from the SAX column and cardiolipin accounts for the remaining $3 \mathrm{~mol} \%$. Each mole of cardiolipin contains 4 mol of esterified fatty acid of which linoleic acid (18:2) comprises over $80 \%$ of the total esterified fatty acid. Accordingly, following transesterification of the SAX eluate in $\mathrm{HCl} / \mathrm{methanol}, \sim 12 \mathrm{~mol} \%$ of the total FAME produced arises from the 18:2 fatty acids potentially esterified to cardiolipin. As shown in Table II, 18:2 fatty acids account for $7-12 \%$ of the total FAME which likely represents fatty acids produced by hydrolysis of the small amount of cardiolipin present in the eluate from the SAX column. Therefore, the contribution of 18:2 FAME was not included in the calculation of the total LPC values.

These results demonstrate a significant increase of LPC in myocardium after a 5-min interval of ischemia. Pretreatment with POCA, $10 \mathrm{~min}$ before the onset of ischemia, prevented the accumulation of LPC and long-chain acylcarnitines and the development of early VT and VF within the 5-min interval of study before acquisition of myocardial samples (Figs. 3-5). 
Table II. Distribution of Fatty Acid Species in LPC

\begin{tabular}{|c|c|c|c|c|c|c|}
\hline & 16:0 & 18:0 & 18:1 & $18: 2$ & Not identified & Sum \\
\hline \multicolumn{7}{|l|}{ Anterior zone } \\
\hline Control $(n=6)$ & $\begin{array}{c}1.57 \pm 0.16 \\
(36 \%)\end{array}$ & $\begin{array}{c}1.22 \pm 0.16 \\
(28 \%)\end{array}$ & $\begin{array}{c}0.54 \pm 0.11 \\
(12 \%)\end{array}$ & $\begin{array}{c}0.52 \pm 0.17 \\
(12 \%)\end{array}$ & $\begin{array}{c}0.48 \pm 0.10 \\
(11 \%)\end{array}$ & $4.33 \pm 0.76$ \\
\hline Ischemia without POCA $(n=5)$ & $\begin{array}{c}3.49 \pm 0.41 \\
(35 \%)\end{array}$ & $\begin{array}{c}2.50 \pm 0.26 \\
(25 \%)\end{array}$ & $\begin{array}{c}1.47 \pm 0.49 \\
(14 \%)\end{array}$ & $\begin{array}{c}1.17 \pm 0.39 \\
(12 \%)\end{array}$ & $\begin{array}{c}1.23 \pm 0.23 \\
(12 \%)\end{array}$ & $9.86 \pm 1.20$ \\
\hline Ischemia with POCA $(n=4)$ & $\begin{array}{c}1.75 \pm 0.22 \\
(37 \%)\end{array}$ & $\begin{array}{c}1.75 \pm 0.35 \\
(37 \%)\end{array}$ & $\begin{array}{c}0.51 \pm 0.21 \\
(11 \%)\end{array}$ & $\begin{array}{c}0.34 \pm 0.10 \\
(7 \%)\end{array}$ & $\begin{array}{c}0.35 \pm 0.11 \\
(7 \%)\end{array}$ & $4.70 \pm 0.82$ \\
\hline \multicolumn{7}{|l|}{ Posterior zone } \\
\hline Control $(n=6)$ & $\begin{array}{c}1.40 \pm 0.15 \\
(36 \%)\end{array}$ & $\begin{array}{c}1.11 \pm 0.11 \\
(28 \%)\end{array}$ & $\begin{array}{c}0.58 \pm 0.11 \\
(15 \%)\end{array}$ & $\begin{array}{c}0.36 \pm 0.08 \\
(9 \%)\end{array}$ & $\begin{array}{c}0.47 \pm 0.08 \\
(12 \%)\end{array}$ & $3.92 \pm 0.69$ \\
\hline Ischemia without POCA $(n=5)$ & $\begin{array}{c}1.98 \pm 0.24 \\
(36 \%)\end{array}$ & $\begin{array}{c}1.52 \pm 0.17 \\
(28 \%)\end{array}$ & $\begin{array}{c}0.73 \pm 0.22 \\
\quad(13 \%)\end{array}$ & $\begin{array}{c}0.68 \pm 0.17 \\
(12 \%)\end{array}$ & $\begin{array}{c}0.55 \pm 0.07 \\
(10 \%)\end{array}$ & $5.46 \pm 0.93$ \\
\hline Ischemia with POCA $(n=4)$ & $\begin{array}{c}1.54 \pm 0.09 \\
(42 \%)\end{array}$ & $\begin{array}{c}1.20 \pm 0.28 \\
(33 \%)\end{array}$ & $\begin{array}{c}0.36 \pm 0.12 \\
(10 \%)\end{array}$ & $\begin{array}{c}0.25 \pm 0.07 \\
(7 \%)\end{array}$ & $\begin{array}{c}0.29 \pm 0.04 \\
(8 \%)\end{array}$ & $3.64 \pm 0.72$ \\
\hline
\end{tabular}

Values are expressed as means \pm SEM in $\mathrm{nmol} / \mathrm{mg}$ protein. The composition of esterified fatty acids in LPC is designated in the form a:b where $a=$ number of carbon atoms and $b=$ number of double bonds.

Hemodynamics. In separate animals $(n=4)$, the influence of POCA on cardiac performance and hemodynamics was evaluated. Two separate 5-min coronary occlusions were implemented with an intercalated 20-min interval of reperfusion. POCA was administered 10 min after reperfusion so that the influence of ischemia with and without inhibition of carnitine acyltransferase I could be delineated in the same animal (Fig. 6). Administration of POCA during the last 10 min of reperfusion did not significantly influence heart rate, cardiac output, stroke volume, systolic, diastolic, or mean blood pressure, or end-systolic or end-diastolic left ventricular pressure. Total peripheral resistance was not altered. The hemodynamic response to induced myocardial ischemia was not altered by treatment with POCA with the sole exception being that left ventricular end-diastolic pressure 1 and $3 \mathrm{~min}$ after the onset of ischemia was 4.8 and $9.8 \mathrm{mmHg}$ lower in animals treated with POCA compared with values in those without POCA. 5 min after the onset of ischemia, left ventricular end-diastolic pressure was similar whether or not POCA had been given (Fig. 6).

Regional myocardial blood flow. To determine whether the antiarrhythmic effect of POCA could have been a reflection of changes in regional myocardial blood flow, radioactively labeled tracer microspheres were administered to four animals subjected to LAD coronary occlusion in the presence and in the absence of POCA. The animals were the same as those in which left ventricular performance and hemodynamics were evaluated. Regional perfusion was measured throughout the entire left ventricle.

Fig. 7 depicts a blood flow map from an animal in which microspheres were injected under each of the following conditions: control, occlusion 1, reperfusion with POCA, and occlusion 2. The control maps, at the left, demonstrate normal, homogeneous blood flow throughout the heart. Control blood flow over 46 endocardial and epicardial zones (including the two papillary muscles) averaged $2.4 \pm 0.1 \mathrm{ml}-\mathrm{min}^{-1}-\mathrm{g}^{-1}$. Blood flow maps obtained during the first coronary occlusion demonstrate a large anterior left ventricular flow deficit involving the septum and apex. Maps obtained during reperfusion and after administration of POCA demonstrate reversal of the flow deficits toward control values with only small areas of residual flow impairment at the apex. Maps obtained during the second occlusion and after administration of POCA exhibit flow patterns virtually identical to those seen during the first occlusion in the absence of POCA. Results in all of the animals were similar.

Blood flow before the first occlusion in each of the four animals is shown in Table III. Mean, control flow for each heart was calculated by averaging blood flow in all $44(n=1)$ or $46(n=3)$ regions of the left ventricle sampled and was found to average $2.44 \pm 0.52 \mathrm{ml}-\mathrm{min}^{-1}-\mathrm{g}^{-1}$ for the four animals. Endocardial blood flow was generally $(n=3)$ higher than epi-
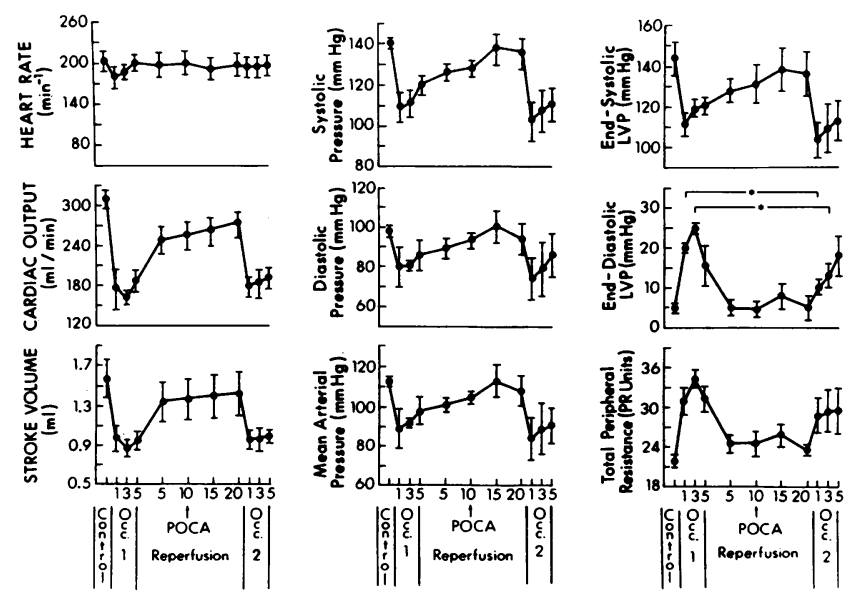

Figure 6. Hemodynamic effects of ischemia with and without treatment with POCA. Measurements of hemodynamic parameters were made at the end of the control period before ischemia (control), at 1 , 3 , and $5 \mathrm{~min}$ after the onset of the first 5-min interval of LAD coronary artery occlusion (Occ. 1), at 5, 10, 15 and 20 minutes after reperfusion (reperfusion), and at 1,3 , and $5 \mathrm{~min}$ after the onset of the second coronary occlusion (Occ. 2). In all animals, POCA was given 10 min after the onset of reperfusion. ${ }^{*} P<0.05$ for comparison of values using a paired Student's $t$ test. 


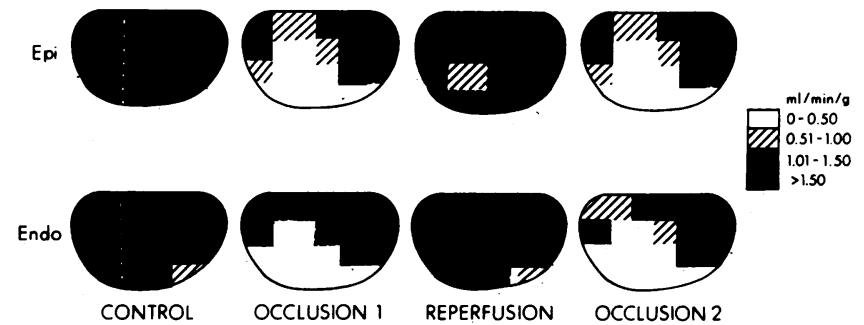

Figure 7. Sequential blood flow maps determined using radiolabeled microspheres from a representative animal in which regional myocardial blood flow was determined before coronary occlusion (control); 4 min after the first coronary occlusion (occlusion 1); $18 \mathrm{~min}$ after reperfusion which was $8 \mathrm{~min}$ after the administration of POCA (reperfusion); and $4 \mathrm{~min}$ after the second coronary occlusion (occlusion 2). Flow values from 22 pieces of epicardium are shown above; flow values from 22 pieces of endocardium are shown below. The left ventricle was cut vertically from base to apex down the posterior septum (left and right borders of each diagram). The dotted lines represent the anterior septum. Various levels of blood flow in ml$\min ^{-1}-\mathrm{g}^{-1}$ are represented by the designs shown in the insert. At the base of the left ventricle the perfusion deficit was more pronounced in the epicardium and the deficit was transmural at the apex and mid-myocardial level.

cardial flow. However, the mean endocardial blood flow to epicardial blood flow ratio was 0.99 .

Flow values obtained during the two intervals of ischemia and with reperfusion were categorized with respect to mean control flow as $0-10 \%, 11-25 \%, 26-50 \%, 51-75 \%$, or $>75 \%$ of the mean, control flow (Fig. 8). The flow deficit produced by the first occlusion resulted in $0-10 \%$ of control flow in $22 \%$ of the left ventricle (LV), $11-25 \%$ in $10 \%, 26-50 \%$ in $15 \%$, $51-75 \%$ in $12 \%$, and $>75 \%$ in $41 \%$ of the LV (Fig. 8 b). Only a modest residual flow deficit was evident with reperfusion after administration of POCA (Fig. $8 \mathrm{c}$ ). Under these conditions flow was greater than $75 \%$ of control in $70 \%$ of the $\mathrm{LV}$ and within one standard deviation of mean, control flow in $91 \%$ of the LV (Fig. $8 c$ ). Flow with the second occlusion, after administration of POCA, (Fig. $8 d$ ) was within $1 \%$ of that with the first occlusion, without POCA, in each of the five tissue flow categories. Thus, POCA did not attenuate the severity of ischemia induced by coronary occlusion. Therefore, the powerful antiarrhythmic effects of inhibition of carnitine acyltransferase I cannot be attributed to amelioration of impaired regional perfusion.

\section{Discussion}

The results obtained indicate that inhibition of carnitine acyltransferase I under conditions of ischemia in vivo prevents the

Table III. Regional Myocardial Blood Flow under Control Conditions

\begin{tabular}{ccccc}
\hline & Endocardium & Epicardium & Endo/Epi Ratio & Total LV \\
\hline 1 & $2.52 \pm 0.14$ & $2.27 \pm 0.12$ & 1.11 & $2.40 \pm 0.10$ \\
2 & $1.51 \pm 0.04$ & $1.37 \pm 0.05$ & 1.10 & $1.44 \pm 0.03$ \\
3 & $2.08 \pm 0.11$ & $1.99 \pm 0.07$ & 1.05 & $2.04 \pm 0.07$ \\
4 & $3.58 \pm 0.17$ & $4.18 \pm 0.20$ & 0.86 & $3.87 \pm 0.14$ \\
Mean & $2.42 \pm 0.44$ & $2.45 \pm 0.61$ & 0.99 & $2.44 \pm 0.52$ \\
\hline
\end{tabular}

Values are expressed as $\mathrm{ml}-\mathrm{min}^{-1}-\mathrm{g}^{-1}$ (means \pm SEM).
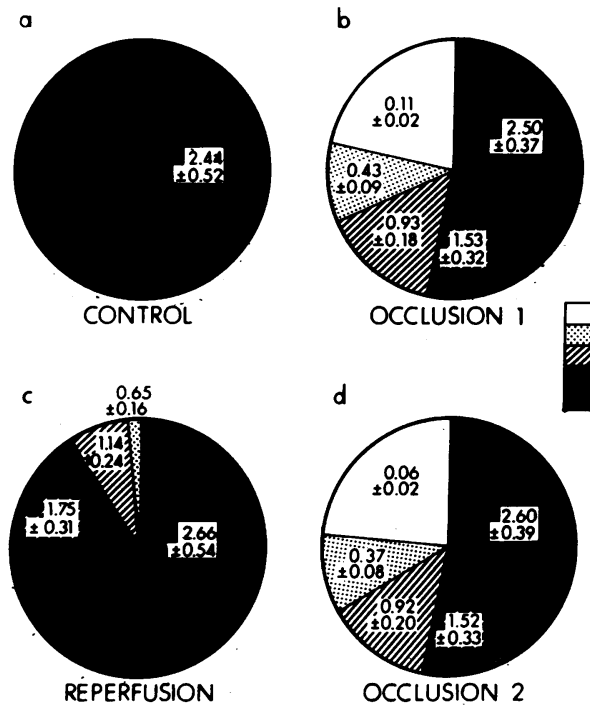

Figure 8. Composite representation of the levels of myocardial blood flow in the left ventricle prior to ischemia ( $a$, control), $4 \mathrm{~min}$ after the first coronary occlusion $(b), 18 \mathrm{~min}$ after reperfusion in the presence of POCA (c), and $4 \mathrm{~min}$ after the second coronary occlusion (d). Each circle represents the entire left ventricle. Each divided segment of the circle represents the fraction of the left ventricle with a given percentage of mean control blood flow as shown in the insert. Numbers within each segment are the mean absolute blood flow values for the corresponding portion of the left ventricle \pm SEM, $n$ $=4$ animals.

accumulation of long-chain acylcarnitines and LPC and confers striking, early antiarrhythmic effects which are not attributable to enhanced regional perfusion or amelioration of impaired hemodynamics. The lack of influence of POCA on the hemodynamic consequences of ischemia have been shown by others as well (34). Although binding of POCA and inhibition of carnitine acyltransferase I is not readily reversible (35), a potential limitation to therapeutic use of this agent in human subjects, the results suggest that development of more specific and reversible inhibitors offers a promising approach to prophylaxis for sudden cardiac death. Agents with a marked increase in inhibitory capacity under conditions occurring in the ischemic heart, such as acidosis, may be particularly useful.

Findings obtained in our laboratory and by others indicate that both long-chain acylcarnitines and lysophosphatides have the capacity to induce electrophysiologic alterations in vitro that resemble changes occurring in the ischemic heart in vivo. Thus (a) tissue is threefold more sensitive to the electrophysiologic effects of exogenous LPC or long-chain acylcarnitines when $\mathrm{pH}$ is reduced to levels seen early after the onset of ischemia in vivo $(3,15)$; $(b)$ LPC increases by $88 \%$ in effluents from ischemic myocardium to a concentration sufficient to induce marked electrophysiologic effects in normoxic cells in vitro when $\mathrm{pH}$ is reduced concomitantly to 6.7 (15); (c) replacement of as little as 1-2 mol\% of myocardial phospholipid or $2 \mathrm{~mol} \%$ of sarcolemmal phospholipid by exogenous amphiphiles, quantified by electron microscopic autoradiography, is sufficient to induce marked electrophysiologic derangements $(17,18) ;(d)$ reversal of the induced electrophysiologic alterations is associated with a decrease in overall tissue content of the amphiphiles resulting from catabolism (17); (e) the electrophysiologic effects of both long-chain acylcarnitines and LPC 
do not require interaction of micelles, monomers are sufficient $(5,6) ;(f)$ LPC can induce delayed afterdepolarizations in isolated tissue even in the presence of acidosis and hyperkalemia, an effect that is augmented markedly by catecholamines (36); $(g)$ the electrophysiologic effects of LPC depend primarily on extracellular delivery into the membrane or sarcolemmal accumulation per se in view of the fact that intracellular delivery with pressure microinjection of high concentrations is without appreciable electrophysiologic effects (16); (h) LPC accumulates within minutes in lymph from the ischemic heart to a concentration sufficient to induce electrophysiologic derangements implicating the extracellular accumulation as critical in arrhythmogenesis (16); (i) LPC accumulates within 2-3 min in ischemic tissue in vivo, and the magnitude of accumulation is reflected by the severity of arrhythmias (37); $(j)$ longchain acylcarnitines accumulate endogenously in isolated myocytes exposed to hypoxia with a marked 70 -fold increase in the sarcolemma judging from results of EM autoradiography of prelabeled $\left[{ }^{3} \mathrm{H}\right]$ carnitine pools $(11) ;(k)$ inhibition of carnitine acyltransferase I not only precludes the sarcolemmal accumulation of long-chain acylcarnitines but also markedly attenuates electrophysiologic derangements otherwise evident in hypoxic myocytes (11). Results of the present study demonstrate that attenuation of accumulation of LPC and long-chain acylcarnitines in vivo results in striking protection against early ventricular fibrillation otherwise induced by ischemia.

Antiarrhythmic effects of inhibition of carnitine acyltransferase I appear to reflect decreased accumulation of long-chain acylcarnitines and LPC. Because long-chain acylcarnitines inhibit two of the enzymes responsible for catabolism of LPC in heart, lysophospholipase, and lysophospholipase-transacylase $(19,20)$, attenuation of the increase of long-chain acylcarnitines in response to ischemia is likely to attenuate the inhibition of catabolism of LPC preventing its accumulation. Although a reduction of $\mathrm{pH}$ inhibits membrane-bound lysophospholipase activity in myocardium (38), the results of the present study suggest that a concomitant increase in the concentration of long-chain acylcarnitines is necessary for inhibition of accumulation of LPC.

The antiarrhythmic effect of POCA may be dependent also on attenuation of the increase in $\alpha_{1}$-adrenergic receptors and their coupling to intracellular processes in ischemic myocytes. We and others have demonstrated potent antiarrhythmic effects of $\alpha_{1}$-adrenergic blockade (for review see reference 14) as well as an increase in $\alpha_{1}$-adrenergic receptors in ischemic myocardium (39-41). Adult canine myocytes exposed to conditions of hypoxia sufficient to induce marked increases in concentrations of long-chain acylcarnitines exhibit a two- to threefold increase in surface $\alpha_{1}$-adrenergic receptors (12). The increase is blocked by inhibition of carnitine acyltransferase I with POCA. It can be induced in normoxic myocytes exposed to low concentrations of exogenous long-chain acylcarnitines (12). The increase in $\alpha_{1}$-adrenergic receptors in hypoxic myocytes is reversible with reoxygenation and is coupled to an increase in $\mathrm{IP}_{3}$ (13). Therefore, a portion of the antiarrhythmic effects of POCA in ischemic myocardium in vivo may involve attenuation of the enhanced $\alpha_{1}$-adrenergic responsivity otherwise apparent when concentrations of long-chain acylcarnitines increase in the sarcolemma.

Inhibition of carnitine acyltransferase I with POCA may enhance the accumulation of another amphiphile, acyl CoA, in ischemic myocardium. However, the magnitude of the in- crease of acyl CoA with ischemia is much smaller than that of long-chain acylcarnitine $(42,43)$. Acyl CoA is confined, almost exclusively, to the mitochondria (44) and is therefore unlikely to impact directly on the sarcolemma particularly early after the onset of ischemia.

The influence of inhibition of carnitine acyltransferase I with POCA on the accumulation of long-chain acylcarnitines and LPC and the concomitant effects on arrhythmogenesis required our obtaining transmural biopsies after relatively short durations of ischemia. Because the biopsy procedure results in a major perturbation of the heart, experiments had to be terminated promptly. It is well known that the incidence of both VT and VF is maximal very early after the onset of ischemia with a precipitous decline thereafter. Accordingly, we focused on the 5-min interval after the onset of ischemia.

Inhibition of carnitine acyltransferase I with POCA decreases extraction of free fatty acids by myocardium (45). However, it is unlikely that this phenomenon contributes substantially to its antiarrhythmic effects. Nonesterified fatty acid concentrations do not increase in ischemic tissue for at least 10 min (33). POCA inhibits the oxidation of fatty acids and increases glucose oxidation 2- to 2.5-fold (46). Enhanced glucose utilization may increase ATP production in ischemic tissue by increasing the extent of anaerobic glycolysis. However, salutary augmentation of ATP content is unlikely judging from the limited, maximal rate of ATP production through anaerobic glycolysis and the lack of attenuation of negative inotropic effects in hearts from treated animals compared with those not treated with POCA. Others have shown that POCA exerts no striking effects on tissue ATP or lactate levels in response to ischemia (47).

Inhibition of cardiac lipoprotein lipase activity by POCA results in increased clearance of triglycerides from plasma (48) and inhibition of synthesis of lipids in the liver (49). Because these effects occur over relatively long intervals, they are unlikely to account for the antiarrhythmic effects seen in our study.

We have recently shown that the arrhythmias occurring within the first few minutes after the onset of myocardial ischemia in vivo in cats involve reentry attributable to slow conduction and variable degrees of transmural conduction block as well as non-reentrant mechanisms judging from analysis of three-dimensional maps obtained when spontaneous ventricular arrhythmias occurred (50). Exogenous LPC and long-chain acylcarnitines result in marked decreases in the resting membrane potential and depression in the $\dot{V}_{\max }$ of phase 0 in vitro (1-6), changes that could mediate the marked slowing of conduction and the variable conduction block predisposing to arrhythmias dependent on reentrant mechanisms. In addition, shortening of the refractory period, seen particularly at low concentrations of the amphiphiles in vitro, would be expected to facilitate the development of reentry (2-4).

The non-reentrant mechanism manifest in ischemic hearts may involve the development of delayed afterdepolarizations associated with an early and rapid increase in cytosolic $\mathrm{Ca}^{2+}$ (10). Incorporation of exogenous LPC into the sarcolemma in isolated tissue leads to an increase in cytosolic $\mathrm{Ca}^{2+}$ and contractility $(6,7)$ but is associated with a decrease in the slow inward current $\left(I_{\mathrm{si}}\right)$ carried by $\mathrm{Ca}^{2+}(6)$. This increased cytosolic $\mathrm{Ca}^{2+}$ is likely to reflect enhancement of $\mathrm{Na}^{+}-\mathrm{Ca}^{+}$exchange secondary to the LPC-induced inhibition of $\mathrm{Na}^{+}-\mathrm{K}^{+}$ ATPase (51) and accumulation of intracellular $\mathrm{Na}^{+}$. Recently, 
we have shown that exogenous LPC can induce delayed afterdepolarizations and triggered rhythms in vitro (36), effects stimulated by catecholamines. Long-chain acylcarnitines can activate calcium channels in cardiac muscle and smooth muscle (7-9). Accordingly, the antiarrhythmic influence of POCA seen in the present study is likely to involve amelioration of both reentrant and non-reentrant mechanisms responsible for arrhythmogenesis in ischemic myocardium.

\section{Acknowledgments}

The authors appreciate the initial helpful discussions from Dr. Maureen Knabb, the technical assistance of Bevery Kekec, and the preparation of the manuscript by Ava Ysaguirre.

Supported in part by National Institutes of Health grant HL-17646, SCOR in Ischemic Heart Disease, and NIH grants HL-28995 and HL-36773.

\section{References}

1. Corr, P. B., R. W. Gross, and B. E. Sobel. 1984. Amphipathic metabolites and membrane dysfunction in ischemic myocardium. Circ. Res. 55:135-154.

2. Corr, P. B., M. E. Cain, F. X. Witkowski, D. A. Price, and B. E. Sobel. 1979. Potential arrhythmogenic electrophysiological derangements in canine Purkinje fibers induced by lysophosphoglycerides. Circ. Res. 44:822-832.

3. Corr; P. B., D. W. Snyder, M. E. Cain, W. A. Crafford Jr., R. W. Gross, and B. E. Sobel. 1981. Electrophysiological effects of amphiphiles on canine Purkinje fibers: Implications for dysrhythmia secondary to ischemia. Circ. Res. 49:354-363.

4. Corr, P. B., D. W. Snyder, B. I. Lee, R. W. Gross, C. R. Keim, and B. E. Sobel. 1982. Pathophysiological concentrations of lysophosphatides and the slow response. Am. J. Physiol. 243:H187-H195.

5. Arnsdorf, M. F., and G. J. Sawicki. 1981. The effects of lysophosphatidylcholine, a toxic metabolite of ischemia, on the components of cardiac excitability in sheep Purkinje fibers. Circ. Res. 49:1630.

6. Clarkson, C. W., and R. E. Ten Eick. 1983. On the mechanism of lysophosphatidylcholine-induced depolarization of cat ventricular myocardium. Circ. Res. 52:543-556.

7. Inoue, D., and A. J. Pappano. 1983. L-Palmitoylcarnitine and calcium ions act similarly on excitatory ionic currents in avian ventricular muscle. Circ. Res. 52:625-634.

8. Spedding, M. 1985. Activators and inactivators of $\mathrm{Ca}^{++}$channels: New perspectives. J. Pharmacol. (Paris). 16:319-343.

9. Spedding, M., and A. K. Mir. 1987. Direct activation of $\mathrm{Ca}^{++}$ channels by palmitoyl carnitine, a putative endogenous ligand. $B r . J$. Pharmacol. 92:457-468.

10. Lee, H. C., N. Smith, R. Mohabir, and W. T. Clusin. 1987. Cytosolic calcium transients from the beating mammalian heart. Proc. Natl. Acad. Sci. USA. 84:7793-7797.

11. Knabb, M. T., J. E. Saffitz, P. B. Corr, and B. E. Sobel. 1986. The dependence of electrophysiological derangements on accumulation of endogenous long-chain acyl carnitine in hypoxic neonatal rat myocytes. Circ. Res. 58:230-240.

12. Heathers, G. P., K. A. Yamada, E. M. Kanter, and P. B. Corr. 1987. Long-chain acylcarnitines mediate the hypoxia induced increase in alpha ${ }_{1}$-adrenergic receptors on adult canine myocytes. Circ. Res. 61:735-746.

13. Heathers, G. P., P. B. Corr, and A. S. Evers. 1987. A novel method for measurement of mass of inositol phosphates in adult canine myocytes. Circulation. 76(Suppl. IV):IV-13.

14. Heathers, G. P., K. A. Yamada, S. M. Pogwizd, and P. B. Corr. 1988. The contribution of $\alpha$-and $\beta$-adrenergic mechanisms in the genesis of arrhythmias during myocardial ischemia and reperfusion. In
Neurocardiology. H. E. Kulbertus, and G. Franck, editors. Futura, Mount Kisco, NY. 143-178.

15. Snyder, D. W., W. A. Crafford, Jr., J. L. Glashow, D. Rankin, B. E. Sobel, and P. B. Corr. 1981. Lysophosphoglycerides in ischemic myocardium effluents and potentiation of their arrhythmogenic effects. Am. J. Physiol. 241:H700-H707.

16. Akita, H., M. H. Creer, K. A. Yamada, B. E. Sobel, and P. B. Corr. 1986. The electrophysiologic effects of intracellular lysophosphoglycerides and their accumulation in cardiac lymph with myocardial ischemia in dogs. J. Clin. Invest. 78:271-286.

17. Gross, R. W., P. B. Corr, B. I. Lee, J. E. Saffitz, W. A. Crafford, Jr., and B. E. Sobel. 1982. Incorporation of radiolabeled lysophosphatidyl choline into canine Purkinje fibers and ventricular muscle: electrophysiological, biochemical, and autoradiographic correlations. Circ. Res. 51:27-36.

18. Saffitz, J. E., P. B. Corr, B. I. Lee, R. W. Gross, E. K. Williamson, and B. E. Sobel. 1984. Pathophysiologic concentrations of lysophosphoglycerides quantified by electron microscopic autoradiography. Lab. Invest. 50:278-286.

19. Gross, R. W., and B. E. Sobel. 1983. Rabbit myocardial cytosolic lysophospholipase. Purification, characterization, and competitive inhibition by L-palmitoyl carnitine. J. Biol. Chem. 258:52215226.

20. Gross, R. W., R. C. Drisdel, and B. E. Sobel. 1983. Rabbit myocardial lysophospholipase-transacylase. Purification, characterization, and inhibition by endogenous cardiac amphiphiles. J. Biol. Chem. 258:15165-15172.

21. Heymann, M. A., B. D. Payne, J. I. E. Hoffman, and A. M. Rudolph. 1977. Blood flow measurements with radionuclide-labeled particles. Prog. Cardiovasc. Dis. 20:55-79.

22. Chen, P. S., Jr., T. Y. Toribara, and H. Warner. 1956. Microdetermination of phosphorous. Anal. Chem. 28:1756-1758.

23. Creer, M. H., C. Pastor, P. B. Corr, R. W. Gross, and B. E. Sobel. 1985. Quantification of choline and ethanolamine phospholipids in rabbit myocardium. Anal. Biochem. 144:65-74.

24. Bligh, E. G., and W. J. Dyer. 1959. A rapid method of total lipid extraction and purification. J. Biochem. Physiol. 37:911-917.

25. Gross, R. W., and B. E. Sobel. 1980. Isocratic high-performance liquid chromatography separation of phosphoglycerides and lysophosphoglycerides. J. Chromatogr. 197:79-85.

26. Gross, R. W. 1984. High plasmalogen and arachidonic acid content of canine myocardial sarcolemma: A fast atom bombardment mass spectroscopic and gas chromatography-mass spectroscopic characterization. Biochemistry. 23:158-165.

27. Williamson, J. R., and B. E. Corkey. 1969. Assays of intermediates of the citric acid cycle and related compounds by fluorometric enzyme methods. In Methods in Enzymology. J. M. Lowenstein, editor. Academic Press, NY. Vol. 8. 437-440.

28. McGarry, J. D., and D. W. Foster. 1976. An improved and simplified radioisotopic assay for the determination of free and esterified carnitine. J. Lipid Res. 17:277-281.

29. Markwell, M. A. K., S. M. Haas, N. E. Tolbert, and L. L. Bieber. 1981. Protein determination in membrane lipoprotein samples: manual and automated procedures. Methods Enzymol. 72:296303.

30. Whitmer, J. T., J. A. Idell-Wenger, M. J. Rovetto, and J. R. Neely. 1978. Control of fatty acid metabolism in ischemic and hypoxic hearts. J. Biol. Chem. 253:4305-4309.

31. Man, R. Y. K., T. L. Slater, M. P. Pelletier, and P. C. Choy. 1983. Alterations of phospholipids in ischemic canine myocardium during acute arrhythmia. Lipids. 18:677-681.

32. Gloster, J., and P. Harris. 1970. The lipid composition of mitochondrial and microsomal fractions of rat myocardial homogenates. Cardiovasc. Res. 4:1-5.

33. Prinzen, F. W., G. J. Van der Vusse, T. Arts, T. H. M. Roemen, W. A. Coumans, and R. S. Reneman. 1984. Accumulation of nonesterified fatty acids in ischemic canine myocardium. Am. J. Physiol. (Heart Circ. Physiol.) 16:H264-H272. 
34. Seitelberger, R., O. Kraupp, M. Winkler, G. Brugger, and G. Raberger. 1985. Effects of the acylcarnitine-transferase blocking agent sodium 2(5-(4-chlorophenyl)-pentyl)-oxirane-2-carboxylate (POCA) on metabolism and regional function in the underperfused canine myocardium. J. Cardiovasc. Pharmacol. 7:273-280.

35. Wolf, H. P. O., and D. W. Engel. 1985. Decrease of fatty acid oxidation, ketogenesis and gluconeogenesis in isolated perfused rat liver by phenylalkyl oxirane carboxylate (B 807-27) due to inhibition of CPT I (EC 2.3.1.21). Eur. J. Biochem. 146:359-363.

36. Pogwizd, S. M., J. R. Onufer, J. B. Kramer, B. E. Sobel, and P. B. Corr. 1986. Induction of delayed afterdepolarizations and triggered activity in canine Purkinje fibers by lysophosphoglycerides. Circ. Res. 59:416-426.

37. Corr, P. B., K. A. Yamada, M. Creer, A. D. Sharma, and B. E. Sobel. 1987. Lysophosphoglycerides and ventricular fibrillation early after onset of ischemia. J. Mol. Cell. Cardiol. 19:45-53.

38. Gross, R. W., and B. E. Sobel. 1982. Lysophosphatidylcholine metabolism in the rabbit heart. Characterization of metabolic pathways and partial purification of myocardial lysophospholipase-transacylase. J. Biol. Chem. 257:6702-6708.

39. Corr, P. B., J. A. Shayman, J. B. Kramer, and R. J. Kipnis. 1981. Increased $\alpha$-adrenergic receptors in ischemic cat myocardium: A potential mediator of electrophysiological derangements. J. Clin. Invest. 67:1232-1236.

40. Dillon, J. S., X. H. Gu, and W. G. Naylor. 1988. Alpha 1 adrenoceptors in ischemic and reperfused myocardium. J. Mol. Cell. Cardiol. 20:725-735.

41. Maisel, A. S., H. J. Motulsky, M. G. Ziegler, and P. A. Insel. 1987. Ischemia- and agonist-induced changes in $\alpha$ - and $\beta$-adrenergic receptor traffic in guinea pig hearts. Am. J. Physiol. 253:1159-1166.

42. Liedtke, A. J., S. Nellis, and J. R. Neely. 1978. Effects of excess free fatty acids on mechanical and metabolic function in normal and ischemic myocardium in swine. Circ. Res. 43:652-661.
43. Shug, A. L., J. H. Thomsen, J. D. Folts, N. Bittar, M. I. Klein, J. R. Koke, and P. J. Huth. 1978. Changes in tissue levels of carnitine and other metabolites during myocardial ischemia and anoxia. Arch. Biochem. Biophys. 187:25-33.

44. Idell-Wenger, J. A., L. W. Grotyohann, and J. R. Neely. 1978. Coenzyme A and carnitine distribution in normal and ischemic hearts. J. Biol. Chem. 253:4310-4318.

45. Hutter, J. F., H. M. Piper, and P. G. Spieckermann. 1983. Inhibition of fatty acid oxidation in intact working rat heart. Hoppe Seyler's Z. Physiol. Chem. 364:1149-1150.

46. Sherratt, H. S. A., S. J. Gatley, T. R. DeGrado, C. K. Ng, and J. E. Holden. 1983. Effects of 2[5(4-chlorophenyl)pentyl]oxirane-2carboxylate on fatty acid and glucose metabolism in perfused rat hearts determined using iodine-125 16-iodohexadecanoate. Biochem. Biophys. Res. Commun. 117:653-657.

47. Paulson, D. J., J. J. Noonan, K. M. Ward, H. S. A. Sherratt, and A. L. Shug. 1986. Effects of POCA on metabolism and function in the ischemic rat heart. Basic Res. Cardiol. 81:180-187.

48. Rogers, M. P. 1987. Effects of 2(5(4-chlorophenyl)pentyl)oxirane-2-carboxylate on lipoprotein lipase, adipose tissue lipolysis and glycerol phosphate acyltransferase in rats. Biochem. Pharmacol. 36:971-972.

49. Vaartjes, W. J., C. G. M. de Haas, and H. P. Haagsmann. 1986. Effects of sodium 2-[5-(4-chlorophenyl)pentyl]-oxirane-2-carboxylate (POCA) on intermediary metabolism in isolated rat-liver cells. Biochem. Pharmacol. 35:4267-4272.

50. Pogwizd, S. M., and P. B. Corr. 1987. Reentrant and nonreentrant mechanisms contribute to arrhythmogenesis during early myocardial ischemia: Results using three-dimensional mapping. Circ. Res. 61:352-371.

51. Karli, J. N., G. A. Karikas, P. K. Hatzipavlou, G. M. Levis, and S. N. Moulopoulos. 1979. The inhibition of $\mathrm{Na}^{+}$and $\mathrm{K}^{+}$stimulated ATPase activity of rabbit and dog heart sarcolemma by lysophosphatidylcholine. Life Sci. 24:1868-1876. 